Gazi University
Journal of Science
http://dergipark.gov.tr/gujs

\title{
Unbounded Vectorial Cauchy Completion of Vector Metric Spaces
}

\author{
Cetin Cemal OZEKEN (10), Cuneyt CEVIK* \\ Department of Mathematics, Faculty of Sciences, Gazi University, 06500 Teknikokullar Ankara, Turkey
}

\author{
Highlights \\ - Unbounded vectorial convergence in vector metric spaces is studied. \\ - Relations between uo-convergence and $u_{d, E}$-convergence are examined. \\ - An unbounded Cauchy completion of any vector metric space is constructed. \\ - A more general completion of vector metric spaces is obtained.
}

\begin{tabular}{l} 
Article Info \\
\hline Received: 09/08/2019 \\
Accepted: 06/03/2020 \\
Keywords \\
Unbounded order convergence \\
Vector metric spaces \\
Unbounded vectorial \\
convergence, \\
Unbounded \\
Cauchy completion, \\
Riesz space
\end{tabular}

\section{INTRODUCTION}

Riesz spaces are partially ordered vector spaces in which structure of the space and partial order is compatible, and for any two elements their supremum exists. A Riesz space $E$ is called Dedekind complete if every nonempty subset of $E$ has a supremum whenever it is bounded from above. For any decreasing sequence $\left(a_{n}\right)$ of $E$, we write $a_{n} \downarrow 0$ if inf $a_{n}=0$. Besides, the Riesz space $E$ is called Archimedean if $n^{-1} a \downarrow 0$ for every $a \in E_{+}$. One of the central tools in Riesz spaces is order convergence. A sequence $\left(a_{n}\right)$ of $E$ is called o-convergent (or order convergent) to $a \in E$ and written as $a_{n} \stackrel{o}{\rightarrow} a$ if there exists a sequence $\left(b_{n}\right)$ in $E$ such that $b_{n} \downarrow 0$ and $\left|a_{n}-a\right| \leq b_{n}$ for all $n$. Every order convergent sequence is order bounded, but this kind of convergence cannot be applied to sequences which are not order bounded. However, there is a more general convergence applicable to not only all bounded but also some unbounded sequences in a Riesz space. A sequence $\left(a_{n}\right)$ in a Riesz space $E$ is called uo-convergent (or unbounded order convergent) to $a \in E$ if $\left|a_{n}-a\right| \wedge u \stackrel{o}{\rightarrow} 0$ for all $u \in E_{+}$. Also, a sequence $\left(a_{n}\right)$ in a Riesz space is called unbounded order Cauchy (or uo-Cauchy) if $\left|a_{n}-a_{n+p}\right|$ is uo-convergent to 0 . It was first defined by H. Nakano in [1] as "individual convergence". Later, R. Demarr [2] used the name "unbounded order convergence" and he showed that one can embed a locally convex space $E$ into a particular ordered vector space in which uoconvergence is equivalent to topological convergence in $E$. Demarr also gave examples indicate the resemblance and difference of o-convergence and uo-convergence. If $E$ is Riesz space of real valued functions defined on a set $X$, o-convergence and uo-convergence coincide if and only if $X$ is finite; moreover, these types of convergence coincide for sequence even if $X$ is infinite. If $E$ is Riesz space of real valued integrable functions defined on $[0,1]$, o-convergence and uo-convergence do not coincide for 
sequences. After that, in Dedekind complete Riesz spaces which have weak units, S. Kaplan constituted two characterizations of uo-convergence [3]. Besides, uo-convergent and uo-Cauchy nets are used in characterization of Banach lattices which have the positive Schur property and KB spaces by N. Gao and F. Xanthos [4]. Later, more properties of uo-convergence are studied by N. Gao, V. Troitsky and F. Xanthos [5]. For other facts regarding Riesz spaces we point to references [6,7].

Vector metric spaces equipped with a Riesz space $E$ valued distance map are defined in [8,9]. A vector metric $d$ on a non-empty set $X$ is a map $d: X \times X \rightarrow E$ satisfying the followings:

$d(x, y)=0$ if and only if $x=y \quad$ and $\quad d(x, y) \leq d(x, z)+d(y, z)$

for all $x, y, z \in X$. The set $X$ or the triple $(X, d, E)$ is called a vector metric space. Also, vectorial convergence is defined in this type of spaces and examined some properties of this convergence. A sequence $\left(x_{n}\right)$ in $X$ vectorially converges (simply $E$-converges) to $x \in X$, which is written as $x_{n} \stackrel{d, E}{\rightarrow} x$, if there exists a sequence $a_{n}$ in $E$ such that $d\left(x_{n}, x\right) \leq a_{n}$ and $a_{n} \downarrow 0$ for all $n$. A nonempty subset $A$ of $X$ is called $E$ bounded if there is a nonzero $a \in E_{+}$such that $d(x, y) \leq a$ for all $x, y \in X$. As the order convergence is not applicable to unbounded order sequences in a Riesz space $E$, the $E$-convergence is also not applicable to sequences which are not $E$-bounded in a vector metric space $X$. In this study, we define unbounded vectorially convergent and unbounded vectorial Cauchy sequences, and examine some properties of them. In addition to that we construct the unbounded Cauchy completion of a given vector metric spaces.

\section{UNBOUNDED VECTORIAL CONVERGENCE}

Throughout this section we assume $X$ as a vector metric space $(X, d, E)$.

Definition 1. Let $\left(x_{n}\right)$ be a sequence in $X$ and $x \in X$. A sequence $\left(x_{n}\right)$ in $X$ is said to be unbounded vectorially convergent (or $u_{d, E^{-}}$-convergent) to $x$ if $d\left(x_{n}, x\right) \wedge u \stackrel{o}{\rightarrow} 0$ for all $u \in E_{+}$. We will denote this vectorial convergence by $x_{n} \stackrel{u_{d, E}}{\longrightarrow} x$.

Definition 2. A sequence $\left(x_{n}\right)$ in $X$ is said to be unbounded $E$-Cauchy (or $u_{E}$-Cauchy) sequence if for every $u \in E_{+}$there exists a sequence $\left(a_{n}\right)$ in $E$ such that $a_{n} \downarrow 0$ and $d\left(x_{n}, x_{n+p}\right) \wedge u \leq a_{n}$ for all $p$ and $n$.

Definition 3. The vector metric space $X$ is said to be $u_{E}$-complete if every $u_{E}$-Cauchy sequence in $X$ is $u_{d, E^{-}}$convergent.

Assume $(X, d, E)$ is a vector metric space, $x \in X$ and $x_{n} \stackrel{u_{d, E}}{\longrightarrow} x$. Then

1. The limit $x$ is unique,

2. Every subsequence of $\left(x_{n}\right)$ is $u_{d, E}$-convergent to $x$,

3. If $x_{n} \stackrel{u_{d, E}}{\longrightarrow} x$ and $y_{n} \stackrel{u_{d, E}}{\longrightarrow} y$ then $d\left(x_{n}, y_{n}\right) \stackrel{u o}{\rightarrow} d(x, y)$.

Moreover, the followings are satisfied.

4. Every $u_{d, E}$-convergent sequence is $u_{E}$-Cauchy sequence,

5. If a $u_{E}$-Cauchy sequence $\left(x_{n}\right)$ has a subsequence $\left(x_{n_{k}}\right)$ such that $x_{n_{k}} \stackrel{u_{d, E}}{\longrightarrow} x$, then $x_{n} \stackrel{u_{d, E}}{\longrightarrow} x$.

6. If $\left(x_{n}\right)$ and $\left(y_{n}\right)$ are $u_{E}$-Cauchy sequences in X, then $d\left(x_{n}, y_{n}\right)$ is uo-Cauchy sequence in $E$.

Now we will give a simple example. 
Example 1. Let $X=\mathbb{R}$ and $E=\mathbb{R}^{2}$. We know that $\mathbb{R}^{2}$ is an Archimedean Riesz space with coordinatwise ordering. Define a function $d: \mathbb{R} \times \mathbb{R} \rightarrow \mathbb{R}^{2}$ as $d(x, y)=\left(\frac{1}{2}|x-y|, \frac{1}{3}|x-y|\right)$. It is easy to see that $(X, d, E)$ is a vector metric space. Let also $\left(x_{n}\right)$ be a sequence whose general term is $x_{n}=\frac{1}{n}$. For any $u \in$ $E_{+}$and for any $n \in \mathbb{N}$, we get $d\left(x_{n}, 0\right) \wedge u \leq\left(\frac{2}{3 n}, \frac{2}{3 n}\right)$. By taking $a_{n}=\left(\frac{2}{3 n}, \frac{2}{3 n}\right)$ we can observe that $\left(a_{n}\right)$ is a sequence in $E$ and $a_{n} \downarrow(0,0)$. Therefore $x_{n} \stackrel{u_{d, E}}{\longrightarrow} 0$.

Now we will give a very important example which emphasize the relation between unbounded vectorial convergence and unbounded order convergence.

Example 2. By taking $X=E$ and $d(x, y)=|x-y|$, unbounded vectorial convergence become simply unbounded order convergence.

The following theorem presents us the relation between vectorial convergence and unbounded vectorial convergence.

Theorem 1. Every vectorial convergent sequence is unbounded vectorial convergent.

Proof. Let $(X, d, E)$ be a vector metric space and $\left(x_{n}\right)$ be a vectorial convergent sequence in $X$. Also let $x_{n} \stackrel{d, E}{\rightarrow} x$. Then there exists a sequence $\left(a_{n}\right)$ in $E$ such that $d\left(x_{n}, x\right) \leq a_{n}$ and $a_{n} \downarrow 0$. Since given $u \in E_{+}$ $d\left(x_{n}, x\right) \wedge u \leq d\left(x_{n}, x\right) \leq a_{n}$ for all $\mathrm{u}$ in $E_{+}$, then we have $x_{n} \stackrel{u_{d, E}}{\longrightarrow} x$.

However, the converse of previous theorem need not to be true.

Example 3. Let $c_{0}$ be the set of real sequences converging to zero, $X=E=c_{0}$ and $d(x, y)=|x-y|$ for all $x, y \in c_{0}$. It is easy to see that vectorial convergence become order convergence and as we have already mentioned unbounded vectorial convergence become unbounded convergence. Suppose that $\left(e_{n}\right)$ is a sequence whose terms are standard unit vectors in $c_{0}$. Since the sequence $\left(e_{n}\right)$ is not ordered bounded, $e_{n} \nrightarrow^{o} 0$ in $c_{0}$. However we can observe that $e_{n} \stackrel{\text { uo }}{\rightarrow} 0$.

Definition 4. Let $X$ be a vector metric space and $Y$ is a nonempty subset of $X$. Then $Y$ is said to be $u_{E}$-dense whenever for every $x \in X$, there exists a sequence $\left(x_{n}\right)$ in $Y$ satisfying $x_{n} \stackrel{u_{d, E}}{\longrightarrow} x$.

A map $f: X \rightarrow Y$ is said to be $E$-isometry if $d(x, y)=\rho(f(x), f(y))$ for all $x, y \in X$ whenever $(X, d, E)$ and $(Y, p, E)$ are vector metric spaces. Besides, $X$ and $Y$ are called $E$-isometric whenever $f$ is onto [8].

\section{UNBOUNDED CAUCHY COMPLETION OF VECTOR METRIC SPACES}

Suppose $(X, d, E)$ is a vector metric space but it is not $u_{E}$-complete. In this section, we are aiming to build a $u_{E}$-complete vector metric space $(\hat{X}, \hat{d}, E)$ of $(X, d, E)$.

Definition 5. Let $(X, d, E)$ be a vector metric space and $(\hat{X}, \hat{d}, E)$ be a $u_{E}$-complete vector metric space. If $X$ is $E$-isometric to a $u_{E}$-dense subset of $\hat{X}$ then $(\hat{X}, \hat{d}, E)$ is called unbounded Cauchy completion (shortly $u_{E}$-Cauchy completion) of $(X, d, E)$.

Theorem 2. Every vector metric space $(X, d, E)$ has a $u_{E}$-completion $(\hat{X}, \hat{d}, E)$.

Proof. First of all, we need to define a map $\varphi: X \rightarrow \hat{X}$ which is an $E$-isometry and $\varphi(X)$ is $u_{E}$-dense in $\hat{X}$. Suppose $E$ is a uo-Cauchy complete Riesz space and also suppose $\left(x_{n}\right)$ and $\left(y_{n}\right)$ are two $u_{E}$-Cauchy sequences in $X$. Now we define an equivalence relation over the set of all $u_{E}$-Cauchy sequences in $X$ as 


$$
\left(x_{n}\right) \sim\left(y_{n}\right) \Leftrightarrow d\left(x_{n}, y_{n}\right) \stackrel{\text { uo }}{\rightarrow} 0
$$

We use the notation $\hat{x}, \hat{y}, \ldots$ for the equivalence classes of these $u_{E}$-Cauchy sequences and $\hat{X}$ for the set of these equivalence classes. Let us define a map $\hat{d}: \hat{X} \times \hat{X} \rightarrow E$ as

$\hat{d}(\hat{x}, \hat{y})=\operatorname{uo-lim} d\left(x_{n}, y_{n}\right)$.

Due to that $\left(d\left(x_{n}, y_{n}\right)\right)$ is a uo-Cauchy sequence whenever $\left(x_{n}\right)$ and $\left(y_{n}\right)$ are $u_{E}$-Cauchy sequences in $X$, and $E$ is uo-Cauchy complete, this map is meaningful. Also it is well defined because uo-limit is unique. Moreover, $(\hat{X}, \hat{d}, E)$ is a vector metric space. By using the fact that for all $x, y \in X$, the constant sequences $\left(x_{n}\right)=(x, x, \ldots)$ and $\left(y_{n}\right)=(y, y, \ldots)$ are $u_{E}$-Cauchy sequences in $X$. Besides, by $(x, x, \ldots) \in \hat{x}$ and $(y, y, \ldots) \in \hat{y}$ we define the map $\varphi: X \rightarrow \hat{X}$ as $\varphi(x)=\hat{x}$. Since

$\hat{d}(\varphi(x), \varphi(y))=\hat{d}(\hat{x}, \hat{y})=\operatorname{uo-lim} d\left(x_{n}, y_{n}\right)=d(x, y)$,

$\varphi$ is an $E$-isometry, and hence $(X, d, E)$ is $E$-isometric to $(\varphi(X), \hat{d}, E)$.

Now we will show that $\varphi(X)$ is $u_{E}$-dense in $\hat{X}$. Let $\left(x_{n}\right) \in \hat{x}$ and, for fixed $n_{0}$ we can consider $\varphi\left(x_{n_{0}}\right)$ as an element of $\varphi(X)$. It is clear that $\varphi\left(x_{n_{0}}\right)$ is the equivalence class generated by the constant sequence $\left(x_{n_{0}}, x_{n_{0}}, \ldots\right)$. For every $n \geq n_{0}$ we can find an integer $p$ such that $n=n_{0}+p$. For every $u \in E_{+}$there is a sequence $\left(a_{n}\right)$ in $E$ such that $\left(a_{n}\right) \downarrow 0$ and

$\hat{d}\left(\varphi\left(x_{n_{0}}\right), \hat{x}\right)=\operatorname{uo-} \lim d\left(x_{n_{0}}, x_{n}\right)=\operatorname{uo-} \lim d\left(x_{n_{0}}, x_{n_{0+p}}\right) \leq a_{n_{0}}$

holds for all $n \geq n_{0}$ with $n=n_{0}+p$ since $\left(x_{n}\right)$ is $u_{E}$-Cauchy. Therefore, we get $\hat{d}\left(\varphi\left(x_{n_{k}}\right), \hat{x}\right) \leq a_{n_{k}}$ for all $k$, in other words, $\varphi\left(x_{n_{k}}\right) \stackrel{u_{d, E}}{\longrightarrow} \hat{x}$.

Now we will show that $(\hat{X}, \hat{d}, E)$ is $u_{E}$-complete vector metric space generated by $(X, d, E)$. Suppose $\left(\hat{x}_{n}\right)$ is a $u_{E}$-Cauchy sequence in $\hat{X}$. Since $\varphi(X)$ is $u_{E}$-dense in $\hat{X}$, we can find $y_{n}$ in $X$ and $\left(a_{n}\right)$ in $E$ with $\hat{d}\left(\varphi\left(y_{n}\right), \hat{x}_{n}\right) \leq a_{n}$ for each $n$. For all $u \in E_{+}$and all $p$ and $n$ we have

$$
\begin{aligned}
d\left(y_{n}, y_{n+p}\right) \wedge u & =\hat{d}\left(\varphi\left(y_{n}\right), \varphi\left(y_{n+p}\right)\right) \wedge u \\
& \leq \hat{d}\left(\varphi\left(y_{n}\right), \hat{x}_{n}\right)+\hat{d}\left(\hat{x}_{n}, \hat{x}_{n+p}\right)+\hat{d}\left(\hat{x}_{n+p}, \varphi\left(y_{n+p}\right)\right) \leq 3 a_{n}
\end{aligned}
$$

Therefore $\left(y_{n}\right)$ is a $u_{E}$-Cauchy in $X$. Let $\hat{y}$ is equivalence class generated by $\left(y_{n}\right)$, since $\varphi\left(x_{n}\right) \stackrel{u_{d, E}}{\longrightarrow} \hat{y}$ we get

$\hat{d}\left(\hat{y}, \hat{x}_{n}\right) \leq \hat{d}\left(\hat{y}, \varphi\left(y_{n}\right)\right)+\hat{d}\left(\varphi\left(y_{n}\right), \hat{x}_{n}\right) \leq 2 a_{n}$

which means $x_{n} \stackrel{u_{d, E}}{\longrightarrow} \hat{y}$. Hence $(\hat{X}, \hat{d}, E)$ is $u_{E}$-complete.

Theorem 3. Completion of a vector metric space is unique.

Proof. We will follow standard procedure, taking two $u_{E}$-completion of $X$ and showing that they are isometrically equivalent to each other. Suppose $(\hat{X}, \hat{d}, E)$ is the completion of $X$ as previously mentioned and $(\tilde{X}, \tilde{d}, E)$ is also $u_{E}$-complete vector metric spaces such that there exists an $E$-isometry $\psi: X \rightarrow \tilde{X}$ with $\psi(X)$ is $u_{E}$-dense in $\widetilde{X}$.

First of all we define a map $f_{0}: \varphi(X) \rightarrow \psi(X)$ as $f_{0}(\varphi(x))=\psi(X)$ for all $x \in X$. It can be easily seen that $\varphi(X)$ and $\psi(X)$ are $E$-isometric. We know that there is a $u_{E}$-Cauchy sequence $\varphi\left(x_{n}\right)$ in $\varphi(X)$ such 
that $\varphi\left(x_{n}\right) \stackrel{u_{d, E}}{\longrightarrow} \hat{x}$ if $\hat{x} \in \hat{X}$. Due to that $f_{0}$ is an $E$-isometry, $\psi\left(x_{n}\right)=\left(f_{0} \circ \varphi\left(x_{n}\right)\right)$ is also an $u_{E}$-Cauchy sequence in $\tilde{X}$. By using $u_{E}$-completeness of $\tilde{X}$, we can say that there exists $\tilde{x}$ in $\tilde{X}$ such that $\psi\left(x_{n}\right) \stackrel{u_{d, E}}{\longrightarrow} \tilde{x}$. Now we can define a map $f: \hat{X} \rightarrow \tilde{X}$ with $f(\hat{x})=\tilde{x}$. As we know, for any $\hat{x}, \hat{y} \in \hat{X}$ there exist sequences $\varphi\left(x_{n}\right)$ and $\varphi\left(y_{n}\right)$ in $\varphi(X)$ such that $\varphi\left(x_{n}\right) \stackrel{u_{d, E}}{\longrightarrow} \hat{x}$ and $\varphi\left(y_{n}\right) \stackrel{u_{d, E}}{\longrightarrow} \hat{y}$. Besides, we can observe that $\psi\left(x_{n}\right) \stackrel{u_{d, E}}{\longrightarrow} f(\hat{x})$ and $\psi\left(y_{n}\right) \stackrel{u_{d, E}}{\longrightarrow} f(\hat{y})$. By using completeness part we get

$\tilde{d}(f(\hat{x}), f(\hat{y}))=\operatorname{uo-} \lim \tilde{d}\left(\psi\left(x_{n}\right), \psi\left(y_{n}\right)\right)=\operatorname{uo-} \lim \hat{d}\left(\varphi\left(x_{n}\right), \varphi\left(y_{n}\right)\right)=\hat{d}(\hat{x}, \hat{y})$

because $f_{0}$ is an $E$-isometry. Finally, we are showing that $f$ is surjective. For any $\tilde{x} \in \tilde{X}$ there exists a $u_{E^{-}}$ Cauchy sequence $\psi\left(x_{n}\right)$ in $\psi(X)$ such that $\psi\left(x_{n}\right) \stackrel{u_{d, E}}{\longrightarrow} \tilde{x}$. Due to that $\varphi(X)$ is $E$-isometric to $\psi(X)$, it is deduced that $\varphi\left(x_{n}\right)$ is a $u_{E}$-Cauchy sequence in $\hat{X}$. Since $\mathrm{f}$ is an $E$-isometry, $f\left(\varphi\left(x_{n}\right)\right) \stackrel{u_{d, E}}{\longrightarrow} f(\hat{x})$ whenever $\hat{x}$ is $\hat{d}$-limit of $\varphi\left(x_{n}\right)$. For each $n$ we have

$\tilde{d}(\tilde{x}, f(\hat{x})) \leq \tilde{d}\left(\tilde{x}, \psi\left(x_{n}\right)\right)+\tilde{d}\left(f(\hat{x}), f\left(\varphi\left(x_{n}\right)\right)\right.$

because $f\left(\varphi\left(x_{n}\right)\right)=\psi\left(x_{n}\right)$. Hence $f(\hat{x})=\tilde{x}$ is satisfied in $\tilde{X}$.

\section{CONFLICTS OF INTEREST}

No conflict of interest was declared by the authors.

\section{REFERENCES}

[1] Nakano, H., "Ergodic theorems in semi-ordered linear spaces", Ann. Math., 49: 538-556, (1948).

[2] DeMarr, R., "Partially ordered linear spaces and locally convex linear topological spaces", Illinois J. Math., 8: 601-606, (1964).

[3] Kaplan, S., “On unbounded order convergence, Real Anal. Exchange”, 23(1): 175-184, (1998-99).

[4] Gao, N. and Xanthos, F., "Unbounded order convergence and application to martingales without probability", J. Math. Anal. Appl., 415(2): 931-947, (2014).

[5] Gao, N., Troitsky, V.G. and Xanthos, F., "Uo-convergence and its applications to cesáro means in Banach lattices", Israel Journal of Math., 220: 649-689, (2017).

[6] Aliprantis, C.D. and Burkinshaw, O., Positive Operators, Springer, Dordrecht, (2006).

[7] Luxemburg, W.A.J. and Zaanen, A.C., Riesz Space I, North-Holland, Amsterdam, The Netherland, (1971).

[8] Çevik, C. and Altun, I., "Vector metric spaces and some properties", Topol. Methods Nonlinear Anal., 34(2): 375-382, (2009).

[9] Çevik, C., Altun, I., Şahin, H. and Özeken, Ç.C., "Some fixed point theorems for contractive mapping in ordered vector metric spaces", J. Nonlinear Sci. Appl., 10 (4): 1424-1432, (2017). 